\title{
Suitability and managerial implications of a Master Surgical Scheduling approach
}

\author{
Jeroen M. van Oostrum • Eelco Bredenhoff • \\ Erwin W. Hans
}

Published online: 27 August 2009

(C) The Author(s) 2009. This article is published with open access at Springerlink.com

\begin{abstract}
Operating room (OR) planning and scheduling is a popular and challenging subject within the operational research applied to health services research (ORAHS). However, the impact in practice is very limited. The organization and culture of a hospital and the inherent characteristics of its processes impose specific implementation issues that affect the success of planning approaches. Current tactical OR planning approaches often fail to account for these issues. Master surgical scheduling (MSS) is a promising approach for hospitals to optimize resource utilization and patient flows. We discuss the pros and cons of MSS and compare MSS with centralized and decentralized planning approaches. Finally, we address various implementation issues of MSS and discuss its suitability for hospitals with different organizational foci and culture.
\end{abstract}

Keywords Master surgical scheduling · Organizational issues · Operating room management · Organizational focus and culture $\cdot$ Health care operations planning and scheduling

\section{Introduction}

Within the relatively small part of the operations research/management science research community that is active in the field of healthcare management (Carter 2002), optimization

J.M. van Oostrum ( $\otimes)$

Econometric Institute, Erasmus School of Economics, Erasmus University Rotterdam, PO Box 1738, 3000 DR Rotterdam, The Netherlands e-mail: j.vanoostrum@erasmusmc.nl

E. Bredenhoff · E.W. Hans

School of Management and Governance, University of Twente, PO Box 217, 7500 AE Enschede, The Netherlands

E. Bredenhoff

e-mail: e.bredenhoff@utwente.nl

E.W. Hans

e-mail: e.w.hans@utwente.nl 
of operating room (OR) department planning and scheduling has always been a popular subject (Dexter et al. 2004; Gerchak et al. 1996; Guinet and Chaabane 2003; Hans et al. 2008; Lamiri et al. 2008). As approximately $60 \%$ of all inpatient admissions consist a visit to an OR department, and various scarce and expensive resources are involved, it is apparent that its efficiency is paramount.

The OR department is often regarded as a production facility with many process uncertainties, like emergencies (Wullink et al. 2007), surgery durations (Strum et al. 2000) and resource availability (McIntosh et al. 2006). It is myopic to focus on just the OR department itself. Its schedule influences processes throughout the hospital (van Oostrum et al. 2008). Also, other departments like the intensive care units (ICUs) and wards pose constraints on the OR schedule that may not be ignored (e.g., bed availability after surgery; Vanberkel and Blake 2007). From an operations research perspective, OR planning and scheduling obviously poses very challenging problems. From the OR manager's perspective, the challenge is to actually implement the resulting tools (Stoop and Wiers 1996). The influence of various stakeholders, with varying degrees of autonomy, is often substantial. In the case of OR scheduling, surgical services (with surgeons), surgery and anesthesia assistants, and anesthesiologists, all have a considerable influence on OR management (Glouberman and Mintzberg 2001a, 2001b; Mintzberg 1997). Intelligent OR planning and scheduling approaches proposed in the literature often fail to account for this, which explains their relatively marginal impact in practice (Harper 2002; Roth and van Dierdonck 1995) and the small number of successful implementations in the literature (Blake and Donald 2002).

As we shall argue, Master Surgical Scheduling (MSS) is a very promising approach from both perspectives. It cyclically executes a master schedule of surgery types, which contains slots for surgery types that recur at least once every cycle (of, say, 4 weeks). In most hospitals and especially clinics, a large part of the case mix in the OR department is recurrent. Such recurrent surgery types can be scheduled in advance in an MSS. From an operations research perspective an MSS can be optimized regarding OR utilization, robustness, overtime, resource conflicts (e.g. limited X-rays), etc. Moreover, it can be drawn up to optimize the inflow into subsequent departments (e.g., ICU, wards). From an OR manager's perspective, the cyclic approach lowers the management burden of making a new schedule every week. It also allows early coordination of personnel and departments involved. More importantly, the medical autonomy is maintained: surgeons remain in charge of selecting patients for particular slots in the schedule. Different MSS approaches have been proposed in the literature (Belien and Demeulemeester 2007; Blake and Donald 2002; Van Houdenhoven et al. 2007c; van Oostrum et al. 2008; Vissers et al. 2005). Nevertheless, various MSS implementation issues have not been dealt with.

The aim of this paper is to assess implementation issues of the MSS approach compared to other planning approaches and to discuss its suitability in hospital organizations with different types of foci and culture. Section 2 first provides an overview of the OR planning and scheduling functions, followed by a discussion of the advantages and disadvantages in practice of planning approaches. Section 3 is a detailed discussion of technical and managerial issues and prerequisites for implementation of an MSS. Section 4 discusses the relation between implementation issues in various types of hospital organizations and cultures. Section 5 discusses the suitability of an MSS to hospitals with different foci and culture. In Sect. 6 we conclude the paper. 


\section{OR planning and scheduling approaches}

In this section we compare and discuss the advantages of an MSS approach to other OR planning approaches. We start in Sect. 2.1 with a brief overview of OR planning and scheduling. In Sect. 2.2 we introduce factors that influence the success of a planning approach. We compare a centralized and decentralized planning approach in Sect. 2.3. In Sect. 2.4 we discuss the potential advantages of an MSS approach.

\subsection{A hierarchical overview of OR planning and scheduling}

This section gives an overview of the various OR planning and scheduling problems, and the solution approaches suggested in the literature. We focus entirely on resource capacity planning and scheduling problems, and discard other managerial areas such as medical planning, material coordination, and financial management. To position the various OR planning and scheduling problems we use the classical hierarchical decomposition of the managerial functions into four levels: strategic, tactical, offline and online operational control (Vissers et al. 2001). The remainder of this section subsequently addresses each level. For a complete overview of the literature concerning OR planning and scheduling we refer to (Denton et al. 2007; Dexter et al. 1999a, 1999b, 2004; Guinet and Chaabane 2003; Hans et al. 2008; McIntosh et al. 2006; Pham and Klinkert 2008; Sier et al. 1997; Van Houdenhoven et al. 2007b; Zhou and Dexter 1998).

\section{Strategic OR planning and scheduling}

To reach organizational goals, the strategic level addresses the dimensioning of core OR resources, such as (inpatient, outpatient, emergency) ORs, personnel, instruments (e.g. X-rays), etc. It also involves case mix planning, i.e. the selection of surgery types, and the determination of the desired patient type volumes (Vissers et al. 2002). Agreements are made with surgical services/specialties concerning their annual patient volumes and assigned OR time. The dimensioning of subsequent departments' resources (e.g. ICUs, ward beds) is also done (Vanberkel and Blake 2007). Strategic planning is typically based on historical data and/or forecasts. The planning horizon is typically a year or more.

\section{Tactical OR planning and scheduling}

The tactical level addresses the usage of the resource on a medium term, typically with a planning horizon of several weeks (Blake and Donald 2002; Wachtel and Dexter 2008). The actual patient demand (e.g. waiting lists, appointment requests for surgery) is used as input. In this stage, the weekly OR time is divided over specialties or surgeons, and elective patients are assigned to days. For the division of OR time, two approaches exist. When a closed block planning approach is used, each specialty will receive a number of OR blocks (usually OR-days). In an (uncommon) open block planning approach, OR time is assigned following the arrival of requests for OR time by surgeons.

On the tactical level, the surgery sequence is usually not of concern. Instead on this level is verified whether the planned elective surgeries cause resource conflicts for the OR, for subsequent departments (ICU, wards), or for required instruments with limited availability (e.g. X-rays). The design of a Master Surgical Schedule is a tactical planning problem. 


\section{Offline operational OR planning and scheduling}

The offline operational level addresses the in advance scheduling of resources and sequencing of activities, typically with a planning horizon of a week (Sier et al. 1997). It encompasses the rostering of OR-personnel, and the add-on scheduling of semi-urgent surgeries in reserved or unused OR-time (Dexter et al. 1999b). In addition, it addresses the sequencing of surgeries (Denton et al. 2007), to prevent critical resource conflicts, e.g. regarding X-rays, instrument sets, surgeons, etc. When there are no emergency ORs, the sequencing of the elective surgeries can also aid in spreading the planned starting times of elective surgeries (which are "break-in moments" for emergency surgeries) in order to reduce the emergency surgery waiting time (Wullink et al. 2007).

\section{Online operational OR planning and scheduling}

The online operational level addresses the monitoring and control of the OR activities during the day. Obviously at this level of control, all uncertainty materializes and has to be dealt with. If necessary, surgeries are rescheduled, or even canceled (Dexter et al. 2004; McIntosh et al. 2006). This is usually done by a day coordinator in the OR department. Emergency surgeries, which have to be dealt with as soon as possible, are scheduled, and emergency OR teams may have to be assembled and dispatched to the first available OR. If there are emergency ORs, these emergency surgeries are dispatched in these ORs. If there are no such ORs, they are scheduled somewhere in the elective surgical schedule.

\subsection{Success factors for planning approaches}

We subsequently discuss several criteria that influence the success of a planning approach: data requirements, resource utilization, robustness, alignment with planning of relevant other departments or resources, autonomy of surgeons, managerial workload, and financial control.

\section{Data requirements}

Intelligent planning approaches are data intensive. A larger amount of data or higher level of detail available generally gives more insight in processes and leads to better predictions (Dexter et al. 2007). On the one hand, the quality of planning potentially increases. On the other hand, a smaller amount of data or a lower level of detail required makes it more likely that these data can be obtained.

\section{Resource utilization}

The OR department is one of the most expensive resources in a hospital. Therefore, planning approaches generally try to maximize the utilization of this department. As we shall argue with the following assessment criterion, utilization by itself is not a good performance indicator-it should always be jointly considered with robustness.

\section{Robustness}

Robustness of a planning approach can have two interpretations: robustness against disruptions and robustness against 'cheating'. The former is the extent in which an approach is 
able to deal with disruptions like emergency arrivals, resource unavailability, overtime, and late cancellations. Moreover, a robust approach should deal with such disruptions on a short term. A high responsiveness to disruption reduces the potential number of affected processes in a hospital.

The latter is the extent in which an approach is able to cope with actors trying to 'trick' the planning in their favor. As an example, a surgeon may request more OR time than he actually needs. A planning approach that can handle both disruptions and prevent actors to cheat the system leads to stable schedules and is thus optimally robust.

Robustness against disruptions is typically obtained by using slack time or slack capacity. For example, in an elective surgery, usually some time is reserved at the end of the regular program to deal with possible disruptions. Obviously, the more robust the program, the more time needs to be reserved, and thus the lower the OR utilization. Consequently, robustness and utilization should always be jointly considered as performance criteria (Van Houdenhoven et al. 2007a).

\section{Alignment with planning of relevant other departments or resources}

An OR planning approach should be aligned with the planning of other relevant departments or resources, such as wards, outpatient clinics, and central sterilization departments. A mismatch between, for instance, the surgical schedules and the scheduling of wards, can seriously affect resource utilization and lead to surgery cancellations.

\section{Autonomy of surgeons}

Healthcare delivery necessitates medical decision making by professionals. This includes decisions on when to deliver care, which are not always based on medical necessity. When this is a medical necessity, a planning approach should incorporate sufficient flexibility to allow for this. Here, the surgeon's autonomy is essential.

\section{Managerial workload}

Many logistical concepts like just-in-time and workload control are focused on reducing system complexity, and thereby the managerial workload. This workload consists of the required effort to make planning decisions and maintain operational control. The required managerial workload is influenced by a planning and scheduling approach.

\section{Financial control}

The more centralized a planning approach, the more it allows financial monitoring and control of the OR department's production. This is particularly important for countries with healthcare systems that apply elements of market competition, such as yard stick competition.

\subsection{Decentralized and centralized planning approaches: advantages and disadvantages}

We discern between centralized and decentralized planning approaches. In a decentralized approach, surgeons decide on the assignment of patients. In a centralized approach, a central planner decides on the eventual division of OR time and assignment of patients. Both approaches require hospital information systems to store data and monitor production. The 
Table 1 Summary of the advantages and disadvantages of decentralized and centralized planning approaches

Decentralized planning approach

Full surgeon autonomy

Requires limited data

Reduces managerial workload at tactical level

Requires intensive online operational control

Results in lack of coordination

Low robustness against cheating, low predictability of patient flows, and low utilization
Centralized planning approach

Little surgeon autonomy

Requires substantial amount of data

Substantial workload at tactical level

Requires online operational control

Good integration of multiple planning processes

High robustness against cheating, high predictability of patient flows, and high utilization

allocation of decision making power should be reflected in and supported by such decision support systems and information systems. In a way these systems offer an implementation of the chosen allocation of decision making power. We compare advantages and disadvantages of the centralized and decentralized planning approaches, using the success factors outlined in Sect. 2.2. The comparison is summarized in Table 1.

A decentralized planning approach offers surgeons full autonomy. It requires very limited data, and reduces the managerial workload at a tactical level. However, the lack of coordination amongst surgeons, and between surgeons and other departments, deteriorates predictability of patient flows, robustness, and resource utilization and necessitates a more intensive online operational control. A decentralized approach is not robust against surgeons trying to 'cheat' the system in their advantage, e.g. by claiming more OR time than actually required, thus leading to lower resource utilization. Finally, although a decentralized planning approach allows for monitoring, it complicates the financial control of the OR department's production.

A centralized planning approach offers little autonomy to surgeons. It requires substantial amounts of data and comes with a substantial workload at a tactical level. The resulting schedules are characterized by high robustness and high utilization. The OR department has to gather and record data of the particularities of all surgeons, patients, and treatments types, and continuously update these data to provide the required process insight to the planner. Since fewer actors are involved in a centralized planning approach, integration with other planning processes within the hospital is easier. Nevertheless, this alignment still requires substantial effort. A major disadvantage of this planning approach is the low autonomy of the surgeons. Surgeons are not allowed to decide when to operate a patient, which might result in surgeons 'cheating' the approach by labeling all patients as urgent. Finally, a centralized planning approach allows for monitoring and financial control.

\subsection{Potential advantages of the MSS approach}

An MSS approach comprises of a schedule of recurrent surgery types, which is cyclically executed. The goal of an MSS approach is to optimize utilization, level the workload, and construct a robust schedule. Patients are assigned to the appropriate slots in this schedule. The planning horizon of the schedule is called the MSS cycle length.

The MSS approach combines advantages of both centralized and decentralized approaches. The main advantages are that it offers the autonomy of medical decision making to surgeons (who may assign patients to slots), while at the same time it yields a high utilization, robustness of schedules, a low degree of required organizational effort at operational level, and offers financial control. Although an MSS approach requires a substantial amount 
of data, it reduces the managerial workload as compared to a non-repetitive centralized planning approach.

The repetitive execution of an MSS approach structures the generally chaotic working practice in OR departments. Hospital departments, such as wards, central sterilization departments, and X-rays, can easily anticipate future demands, thereby reducing the required slack at these departments and improving their efficiency. An MSS thus supports alignment of these resources with the OR department.

An MSS offers all the advantages of a centralized planning approach, regarding optimization of resource utilization and workload leveling. Clustering surgery types with a high variability in a single operating room, for example, might be beneficial for reducing overtime (Hans et al. 2008), and enables management to predict surgery start times more accurately. Furthermore, surgery types that require movable resources such as X-rays can be clustered together in one OR to reduce waiting time for such equipment. Consequently, MSS reduces the managerial burden of operational control.

\section{MSS implementation steps}

We present seven steps for implementation of an MSS approach (see also Fig. 1) and address potential issues that might be encountered.

\subsection{Scope of the MSS}

The first step is to define the scope, i.e., the resources and organizational units to be included in the MSS. These are typically the expensive and scarce resources for which increased utilization is beneficial. As an example, an MSS could also cover ICUs, wards, and medical

Fig. 1 Seven steps to implement a Master Surgical Schedule

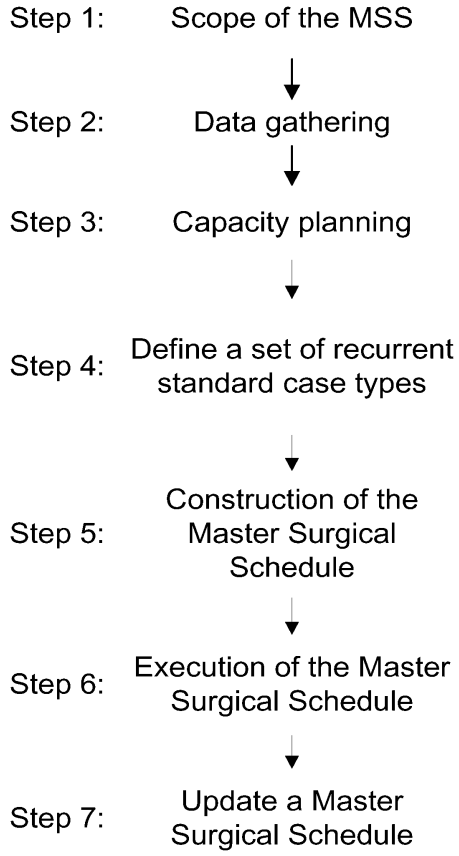


departments. Including a resource or unit is only beneficial if it results in an improved patient flow.

\subsection{Data gathering}

Planning and scheduling relies heavily on reliable data (Harper 2002). Developing an MSS requires at least a year of historical data concerning all processes and resources within its scope. Hospitals routinely collect substantial amounts of process data (Harper 2002). However, these data are often incomplete and polluted, which complicates logistical analyses. Hospitals carefully must record data following strict guidelines, and tools should be implemented that allow quick information retrieval from the involved databases. Detailed and reliable data allow analysis of variance, which is essential for robust planning.

\subsection{Capacity planning}

Based on historical data and trends, capacity plans are made for every resource within the MSS scope. Capacity planning involves resource dimensioning and allocation within the constraints set by target production agreements, and agreements on utilization and availability of resources (Van Houdenhoven et al. 2007a). It also involves reserving slack time for dealing with the inherent variability of the process. There is a trade-off between utilization and robustness: reserving much slack time results in low utilization and high robustness against disruptions and overtime. To determine an acceptable amount of slack per resource, we recommend analyzing the effects of different capacity plans by 'what if' scenarios. Capacity plans should be adjusted accordingly.

Resources are allocated to specialists, specialties, or considered shared. The chosen type of allocation has consequences for the implementation of an MSS. Aside from the technical difficulties that arise when allocating to specialists, sharing resources might invoke resistance. As in most professional organizations, such as hospitals, professionals are organized in groups that are typically outside the control of the organization (Georgopoulos and Mann 1962). This leads to distrust between specialists and managers regarding each other's intentions (Glouberman and Mintzberg 2001a, 2001b). Sharing resources, without clear allocation criteria, might lead to specialists refusing to cooperate. This may delay or even prevent implementation of an MSS.

\subsection{Define a set of recurrent standard case types}

An MSS is built up by a set of recurrent surgery types. Clustering techniques are used on the elective case mix (Bagirov and Churilov 2003; Maruster et al. 2002) to create a limited number of logistically and medically homogeneous surgery types. Examples of logistical characteristics are length of stay and surgery duration; examples of medical characteristics are diagnosis related groups and procedure codes. By periodically updating the set of recurrent surgery types (see Sect. 3.7), a hospital accounts for trends in data, for example seasonality in patient arrival and waiting lists. Aside from the standard surgery types for elective care, additional types are defined to cover emergency and semi-urgent patient mix.

The frequency of a surgery type in the MSS depends on the definition of the surgery type: the broader its definition, the less homogeneous it is but the higher its frequency will be. In addition, the broader the definition of the surgery type, the more the realization of the MSS will deviate from the initial schedule. The definition of the surgery types is thus a trade-off. We suggest the following iterative approach. First, given the initial definition 
of surgery types, each surgery type's frequency is calculated from historical demand and production data. These frequencies are proportionally adjusted to the MSS cycle length, and rounded down. The remaining fractions of surgery types are clustered (based on medical and logistical properties) into a small number of broad defined/dummy surgery types. Ideally, only a small proportion of the case mix is covered by these broad defined surgery types. If their proportion is too large, the definition of the initial surgery types is broadened, and the procedure is repeated. This approach should be repeated for different appropriate MSS cycle lengths to reach an optimum.

The organization may impose restrictions on the surgery type clustering. Management may e.g. impose that the MSS cycle length is aligned with the cycle of the roster of the involved personnel. Also, surgery type clustering is complicated if it takes place on the level of individual surgeons, instead of specialties.

\subsection{Construction of the Master Surgical Schedule}

The surgery types for emergency, semi-urgent and elective care are scheduled in an MSS such that the workload of involved resources is leveled, utilization is optimized, overtime is minimized, and emergency and semi-urgent surgery type waiting time is minimized (Van Houdenhoven et al. 2007c; van Oostrum et al. 2008), subject to various hard and soft constraints. Too many soft constraints that stem from personal preferences impact the quality of the resulting schedule. We refer to van Oostrum et al. (2008) for an advanced approach for constructing an MSS.

\subsection{Execute the Master Surgical Schedule}

For the execution of an MSS, a hospital should develop operational scheduling rules for three groups of patients, i.e., (1) emergency patients, (2) semi-urgent patients, and for patients who require elective surgery (3). Scheduling of emergency patients (1) in an MSS approach is no different than in other scheduling approaches, although we recommend not using a dedicated emergency OR (Wullink et al. 2007). Regarding scheduling rules for semi-urgent patients (2) we suggest scheduling patients based in an earliest due date sequence (Dexter et al. 1999a). Elective surgeries (3) can be planned by either surgeons or by an administrative department. In the latter case, input regarding the medically safe waiting interval is required. Assignment of elective patients is subject to the following guidelines:

- Assignment is allowed within the patient's medically safe interval and the planning horizon;

- Patients are assigned to the most appropriate surgery type slot, on a first come-first serve basis, and as early as possible;

- Re-assigning patients is not allowed during the time required to make preparations for surgery (e.g. material coordination).

The selection of the length of the planning horizon is a trade-off between utilization and waiting time. The longer the planning horizon, the higher the utilization might become, since more possibilities for assigning patients to appropriate slots exist. However, some patients' waiting times might become longer than acceptable since they are postponed to surgery types at the end of the planning horizon, while they would have been assigned to dummy surgery types in case of a short planning horizon. 


\subsection{Update a Master Surgical Schedule}

As long as the access times for all surgery types are balanced, the MSS needs no revision. However, access times change continuously under influence of seasonality in demand and changes in case mix characteristics such as new surgery types. The access times thus need to be monitored regularly, and the MSS should be revised if necessary. Performing a revision of an MSS holds all previous steps, with the only exception that the current MSS is taken as a start. Deviation from the previous MSS is to be minimized to reduce organizational effort related to an MSS update.

\section{Dealing with implementation problems: an organizational approach}

Hospitals consist of organizational units that use both bureaucratic and professional forms of control (Georgopoulos and Mann 1962; Lawrence and Dyer 1983). The organizational culture and focus of these units influence many aspects of MSS implementation.

Organizational culture concerns norms, beliefs, and values (Peterson 1979). In other words, it concerns the mindset of people working in an organization. Four different mindsets or worlds are distinguished in hospitals: care, cure, control, and community (Glouberman and Mintzberg 2001a, 2001b). Distrust amongst these four worlds makes health care a disconnected process.

Organizational focus of a unit/department depends on the patient groups treated and the treatments offered (Davidow and Uttal 1989; Herzlinger 1997). A focused unit is aimed at a limited number of patient groups and/or treatments. Next to general purpose units, we distinguish three types of focused units: specialty based, delivery based, and procedure based. In this section we describe the effects of each of these foci on the organizational culture and how prerequisites of implementing an MSS arise in each of these foci.

\subsection{Specialty based focused units}

Specialty based departments/units are aimed at a single patient group and offer almost all treatments for this group of patients. Examples in the literature are centers for cardiac care and centers for orthopedics (Casalino et al. 2003; Herzlinger 1997; Meyer 1998). These centers encompass all resources required for treatment. As resources in specialty based focused units are dedicated, surgeons have more control (Casalino et al. 2003). We therefore expect organizational culture to highly value professional autonomy.

Successful implementation of MSS in specialty based focused units is also highly dependent on the degree of sub-specialization of surgeons, the volume of patients treated, and the case-mix. A high degree of specialization of surgeons, or high variety in case-mix, complicates defining surgery types, and makes implementing an MSS difficult. For such specialty based organizational units with high sub-specialization and high case-mix variety an MSS such as defined by Belien and Demeulemeester (2007) is more suitable. In general, an MSS can be successfully implemented in specialty based focused units with low sub specialization and in those units where a high sub specialization is combined with large patient volumes and moderate case mix variety.

\subsection{Delivery based focused units}

Delivery based organizational units are aimed at multiple patient groups, which require a similar type of treatment or type of care delivery. The main examples in the literature are 
ambulatory surgery centers (ASCs; Casalino et al. 2003; McLaughlin et al. 1995; Yang et al. 1992). ASCs offer ambulatory, or day-case, surgery and impose limitations on the maximum length of stay, complexity of the surgical procedures, and physical condition of patients. ASCs typically encompass both ORs and wards.

Delivery based focused units exhibit a culture of (strong) common goals related to the timeliness of the care delivery process (Yang et al. 1992). For instance, the authors witnessed remarkable differences in punctuality of starting times of ORs between general purpose and delivery based focused units within a single hospital. While the delivery based (ASC) ORs almost always start on time, the general purpose ORs are on average $20 \mathrm{~min}$ late. These delays can not be explained by emergency or urgent cases. We argue that the difference in average starting time is the consequence of differences in organizational culture.

As multiple patient groups are treated, multiple specialties work in a delivery based unit. The unit's resources, therefore, must be shared between specialties. An MSS offers clear allocation criteria, and guarantees timely and sufficient capacities. In combination with the common goals in this type of unit, this stimulates cooperation between surgeons and personnel. Moreover, since delivery based focused units treat high volumes of patients with low case-mix variety, they are well suited for an MSS approach.

\subsection{Procedure based focused units}

Procedure based focused units are aimed at a single patient group and a single type of treatment. The best known example in the literature of a procedure based organization is the Shouldice Hospital in Canada. Shouldice is aimed at the surgical treatment of hernia patients, and has adapted its processes and lay-out to the patient group treated (Heskett 1983). Examples in the literature of procedure focused organizational units are opthalmologic centers for cataract surgery (Schrijvers et al. 2002).

Procedure based focused units exhibit an even stronger culture of common goals and values than delivery based units. Procedure based focused units treat large volumes of patients, with low case-mix variety and are, therefore, well-suited for MSS.

\subsection{General purpose units}

General purpose units are not aimed at specific patient groups or treatments. Typical examples are the OR department of a community general hospital, or a general ward. These units operate as shared resources, used by all specialties. Resource sharing might lead to conflicts between the four worlds in hospital care. Specialists might be tempted to cheat the system in their advantage, to ascertain required resources. The organizational culture is presumably weak. Whether an MSS approach is feasible for such general purpose units, therefore, strongly depends on case-mix variety, volume, the number of specialties, and the potential efficiency gain. MSS may offer allocation criteria to win support from surgeons, staff and management by creating insights in resource allocation and hereby offer financial control.

\section{Discussion}

Aside from general purpose organizational units, we discussed three different foci for organizational units and their effects on implementing an MSS approach. Hence, the suitability of MSS is affected by the organizational focus and culture, and the case mix characteristics that come with it. 
We argued that implementing an MSS in a hospital consisting of focused organizational units with a low sub specialization of surgeons is relatively easy for a number of reasons. First, focused units exhibit a culture of common norms and beliefs. This culture enforces the willingness to cooperate between specialties, surgeons and personnel, and lowers distrust. Second, delivery and procedure based focused units treat larger numbers of similar patients. As a consequence, repetition is higher, which simplifies defining surgery types. Third, MSS offers clear allocation criteria for shared resources. Last, focused organizational units are, typically, easier to control. Focus thus reduces the managerial workload. Therefore, managerial attention can be paid to implementation of the MSS.

Hospitals with unfocused organizational units that want to implement an MSS might contemplate creating focused organizational units. Focus influences case-mix and volume characteristics of these units, and might thus improve the potential for the MSS approach. Creating such an organizational structure, although requiring considerable efforts, might also further reduce the implementation prerequisites considering managerial control and data collection.

Even implementation of a planning approach that is well-equipped to deal with the characteristics of processes, cultures, and foci in hospitals is often hard. A considerable amount of leadership is required to overcome resistance from surgeons and staff against organizational changes. However, integration of the four worlds (Glouberman and Mintzberg 2001a, 2001b) and alignment of processes of different units potentially improves efficiency (Harper 2002) to the extent that hospitals, in these times of costs reduction, have no other choice.

\section{Conclusions}

We have argued that an MSS approach incorporates the advantages of both decentralized and centralized approaches. The required medical autonomy of surgeons is provided, while due to the in-advance alignment of resources inside and outside the OR high efficiency and robustness are obtained. Due to its repetitive character, an MSS approach minimizes the week-to-week variation in OR production and therefore the resulting demand for other hospital resources. This improves clarity and predictability of work processes, levels workflow, and optimizes patient flows.

We recommend all hospitals to consider implementation of an MSS approach. Hospitals that consist of focused units will benefit most from the advanced planning methods. Hospitals that not yet consist of focused units benefit from MSS implementation through the focusing that comes with the MSS concept. Implementation of an advanced planning approach such as MSS comes with several implementation issues. Particularly the availability of reliable data and weak cooperation between different actors in a hospital organization are of concern. The magnitude of the implementation issues depend on organizational foci and culture, and the inherent characteristics of the processes. We addressed these issues and offered guidelines in this paper for dealing with them.

Open Access This article is distributed under the terms of the Creative Commons Attribution Noncommercial License which permits any noncommercial use, distribution, and reproduction in any medium, provided the original author(s) and source are credited.

\section{References}

Bagirov, A. M., \& Churilov, L. (2003). An optimization-based approach to patient grouping for acute healthcare in Australia. In Lecture notes in computer science: Vol. 2659. Computational science-Iccs 2003 (pp. 20-29). Berlin: Springer. Pt III, Proceedings. 
Belien, J., \& Demeulemeester, E. (2007). Building cyclic master surgery schedules with leveled resulting bed occupancy. European Journal of Operational Research, 176(2), 1185-1204.

Blake, J. T., \& Donald, J. (2002). Mount Sinai hospital uses integer programming to allocate operating room time. Interfaces, 32(2), 63-73.

Carter, M. (2002). Diagnosis: mismanagement of resources. OR/MS Today, 29(2), 26-32.

Casalino, L. P., Devers, K. J., \& Brewster, L. R. (2003). Focused factories? Physician owned specialty facilities. Health Affairs, 22(6), 56-67.

Davidow, W. H., \& Uttal, B. (1989). Service companies: focus or falter. Harvard Business Review, 67, 75-85.

Denton, B., Viapiano, J., \& Vogl, A. (2007). Optimization of surgery sequencing and scheduling decisions under uncertainty. Health Care Management Science, 10, 13-24.

Dexter, F., Epstein, R. H., Traub, R. D., \& Xiao, Y. (2004). Making management decisions on the day of surgery based on operating room efficiency and patient waiting times. Anesthesiology, 101(6), 14441453.

Dexter, F., Macario, A., \& Traub, R. D. (1999a). Optimal sequencing of urgent surgical cases. Journal of Clinical Monitoring and Computing, 15(3-4), 153-162.

Dexter, F., Macario, A., \& Traub, R. D. (1999b). Which algorithm for scheduling add-on elective cases maximizes operating room utilization? Use of bin packing algorithms and fuzzy constraints in operating room management. Anesthesiology, 91(5), 1491-1500.

Dexter, F., Xiao, Y., Dow, A. J., Strader, M. M., Ho, D., \& Wachtel, R. E. (2007). Coordination of appointments for anesthesia care outside of operating rooms using an enterprise-wide scheduling system. Anesthesia and Analgesia, 105(6), 1701-1710.

Georgopoulos, B. S., \& Mann, F. C. (1962). The community general hospital. New York: Macmillan \& Co.

Gerchak, Y., Gupta, D., \& Henig, M. (1996). Reservation planning for elective surgery under uncertain demand for emergency surgery. Management Science, 42(3), 321-334.

Glouberman, S., \& Mintzberg, H. (2001a). Managing the care of health and the cure of disease-Part I: Differentiation. Health Care Management Review, 26(1), 56-69.

Glouberman, S., \& Mintzberg, H. (2001b). Managing the care of health and the cure of disease-Part II: Integration. Health Care Management Review, 26(1), 70-84.

Guinet, A., \& Chaabane, S. (2003). Operating theatre planning. International Journal of Production Economics, 85(1), 69-81.

Hans, E., Wullink, G., Van Houdenhoven, M., \& Kazemier, G. (2008). Robust surgery loading. European Journal of Operational Research, 185(3), 1038-1050.

Harper, P. R. (2002). A framework for operational modelling of hospital resources. Health Care Management Science, 5, 165-173.

Herzlinger, R. E. (1997). Market driven healthcare, who wins, who loses in the transformation of America's largest service industry. Reading: Addison-Wesley.

Heskett, J. L. (1983). Shouldice hospital limited. Boston: Harvard Business School Publishing.

Lamiri, M., Xie, X. L., Dolgui, A., \& Grimaud, F. (2008). A stochastic model for operating room planning with elective and emergency demand for surgery. European Journal of Operational Research, 185(3), 1026-1037.

Lawrence, P. R., \& Dyer, D. (1983). Renewing American industry. New York: Free Press.

Maruster, L., Weijters, T., de Vries, G., van den Bosch, A., \& Daelemans, W. (2002). Logistic-based patient grouping for multi-disciplinary treatment. Artificial Intelligence in Medicine, 26(1-2), 87-107.

McIntosh, C., Dexter, F., \& Epstein, R. H. (2006). The impact of service-specific staffing, case scheduling, turnovers, and first-case starts on anesthesia group and operating room productivity: a tutorial using data from an Australian hospital. Anesthesia and Analgesia, 103(6), 1499-1516.

McLaughlin, C. P., Yang, S., \& van Dierdonck, R. (1995). Professional service organizations and focus. Management Science, 41, 1185-1193.

Meyer, H. (1998). Focused factories. Hospital \& Health Networks, 72(7), 24-30.

Mintzberg, H. (1997). Toward healthier hospitals. Health Care Management Review, 22(4), 9-18.

Peterson, R. A. (1979). Revitalizing the culture concept. Annual Review of Sociology, 5, 137-166.

Pham, D. N., \& Klinkert, A. (2008). Surgical case scheduling as a generalized job shop scheduling problem. European Journal of Operational Research, 185(3), 1011-1025.

Roth, A. V., \& van Dierdonck, R. (1995). Hospital resource planning: concepts, feasibility, and framework. Production and Operations Management, 4(1), 2-29.

Schrijvers, G., Oudendijk, N., \& de Vries, P. (2002). Moderne patiëntenzorg in Nederland: Van kennis naar actie. Amsterdam: Reed Business BV.

Sier, D., Tobin, P., \& McGurk, C. (1997). Scheduling surgical procedures. Journal of the Operational Research Society, 48(9), 884-891.

Stoop, P. P. M., \& Wiers, V. C. S. (1996). The complexity of scheduling in practice. International Journal of Operations \& Production Management, 16(10), 37. 
Strum, D. P., May, J. H., \& Vargas, L. G. (2000). Modeling the uncertainty of surgical procedure times: comparison of log-normal and normal models. Anesthesiology, 92(4), 1160-1167.

Van Houdenhoven, M., Hans, E. W., Klein, J., Wullink, G., \& Kazemier, G. (2007a). A norm utilisation for scarce hospital resources: evidence from operating rooms in a dutch university hospital. Journal of Medical Systems, 31(4), 231-236.

Van Houdenhoven, M., van Oostrum, J. M., Hans, E. W., Wullink, G., \& Kazemier, G. (2007b). Improving operating room efficiency by applying bin-packing and portfolio techniques to surgical case scheduling. Anesthesia and Analgesia, 105(3), 707-714.

Van Houdenhoven, M., van Oostrum, J. M., Wullink, G., Hans, E. W., Hurink, J. L., Bakker, J., \& Kazemier, G. (2007c). Fewer intensive care unit refusals and a higher capacity utilization by using a cyclic surgical case schedule. Journal of Critical Care, 23, 222-226.

van Oostrum, J. M., Van Houdenhoven, M., Hurink, J. L., Hans, E. W., Wullink, G., \& Kazemier, G. (2008). A master surgical scheduling approach for cyclic scheduling in operating room departments. OR Spectrum, 30(2), 355-374.

Vanberkel, J. T., \& Blake, J. T. (2007). A comprehensive simulation for wait time reduction and capacity planning applied in general surgery. Health Care Management Science, 10, 373-385.

Vissers, J. M. H., Adan, I. J. B. F., \& Bekkers, J. A. (2002). Patient mix optimisation in hospital admission planning: a case study. International Journal of Operations and Production Management, 22, 445-461.

Vissers, J. M. H., Adan, I. J. B. F., \& Bekkers, J. A. (2005). Patient mix optimisation in cardiothoracic surgery planning: a case study. IMA Journal of Management Mathematics, 16(3), 281-304.

Vissers, J. M. H., Bertrand, J. W. M., \& de Vries, G. (2001). A framework for production control in health care organizations. Production Planning \& Control, 12(6), 591-604.

Wachtel, R. E., \& Dexter, F. (2008). Tactical increases in operating room block time for capacity planning should not be based on utilization. Anesthesia and Analgesia, 106(1), 215-226.

Wullink, G., Van Houdenhoven, M., Hans, E. W., van Oostrum, J. M., van der Lans, M., \& Kazemier, G. (2007). Closing emergency operating rooms improves efficiency. Journal of Medical Systems, 31(6), 543-546.

Yang, S., McLaughlin, C. P., Vaughan, R. W., \& Aluise, J. J. (1992). Factory focus in hospital-owned ambulatory surgery. International Journal of Service Industry Management, 3(4), 63-75.

Zhou, J. S., \& Dexter, F. (1998). Method to assist in the scheduling of add-on surgical cases-Upper prediction bounds for surgical case durations based on the log-normal distribution. Anesthesiology, 89(5), 1228-1232. 